\title{
Low-Latency Non-predictive Handover Scheme in Mobile IPv6 Environments
}

\author{
Geunhyung Kim ${ }^{1}$ and Cheeha $\mathrm{Kim}^{2}$ \\ 1 Technology Network Laboratory, Korea Telecom (KT), \\ 463-1 Jeonmin-dong, Yusung-gu, Daejeon, 305-811, Korea, \\ geunkim@kt.co.kr \\ 2 Department of Computer Science and Engineering, \\ Pohang University of Science and Technology(POSTECH), \\ San 31 HyoJa-Dong, Nam-Gu, Pohang, 790-784, Korea, \\ chkim@postech.ac.kr
}

\begin{abstract}
In IP-based wireless/mobile networks, minimizing handover latency is one of the most important issues. Fast handover mobile IPv6 (FMIPv6) reduces the handover latency by handover prediction with link-layer information. It requires additional overhead in terms of signaling and may waste resources by incorrect prediction. In this paper, we introduce a new non-predictive handover scheme which employs a handover agent to speedup handover. Signaling and packet delivery costs associated with handover are introduced to compare the performance of the proposed scheme with FMIPv6 and MIPv6. As a result, we found that the proposed scheme guarantees lower handover latency as well as fewer signaling messages than FMIPv6 regardless of prediction accuracy.
\end{abstract}

\section{Introduction}

Mobile IPv6 (MIPv6) [1] is a protocol aimed at maintaining network connectivity for hosts roaming across the Internet. This protocol provides unbroken connectivity to IPv6 mobile hosts (MHs) using a Care-of-Address (CoA) specific to the point of network attachment, when MHs move from one access point (AP) to another in a different subnet. In MIPv6, although an MH configures its CoA when it roams to a new subnet, the $\mathrm{MH}$ cannot receive IP packets on its current point of attachment until the handover procedures finish. These handover procedures include a movement detection, an address configuration, and a location update. These procedures have been shown to result in a long handover latency.

In most cases, long handover latency strongly degrades the IPv6 packet stream of MHs. Two ways of overcoming this problem are to use hierarchical schemes and to apply techniques known as fast handover. Hierarchical schemes focus on reducing the latency caused by the location update procedure. As an example of hierarchical schemes, hierarchical MIPv6 (HMIPv6) 2] reduces the handover latency by minimizing the location update signaling depth. Fast handover schemes focus on reducing the latency caused by movement detection or address configuration procedures. For example, FMIPv6 [3] reduces the handover 
latency by allowing an $\mathrm{MH}$ to pre-configure a new CoA before it moves to a new network.

Although FMIPv6 can provide fast handover in wireless IP networks by handover prediction based on link-layer information, it requires a higher signaling overhead than MIPv6. Also, FMIPv6 is based on the prediction made by using link-layer information of future events. As a result, this prediction may sometimes be wrong. In addition, the allocated buffer space constitutes useless overhead if the prediction is wrong since packet forwarding for smooth handover is performed in accordance with prediction.

To overcome these problems of FMIPv6, we propose a non-predictive handover scheme that can reduce the handover latency of MHs by minimizing movement detection and address configuration latencies. This paper focuses on the AP assisted handover scheme to reduce the latency caused by movement detection and duplicate address detection (DAD) procedures without handover prediction. To perform movement detection as soon as L2 handover finishes, our scheme uses link-layer state information that represents the establishment and release of L2 association between an $\mathrm{MH}$ and an AP. In addition, to perform movement detection procedure and DAD procedure at once, we introduce a new functional entity named as the fast handover agent (FHA) which retains a Router Advertisement $(R A)$ cache and a neighbor list of neighbors on the link. The $R A$ cache and the neighbor list are used for rapid movement detection and DAD, respectively. Since the FHA holds an $R A$ cache and a neighbor list, it gives information on the default router and whether MH's link-local address to be used on the link conflicts with others. In the proposed scheme, we define a DAD flag in an RA message to indicate if MH's new CoA to be generated conflicts with others. Therefore, an $\mathrm{MH}$ can detect if its address conflicts with others when it receives an RA message from the FHA.

In this paper, we analyze the handover latency with a timing diagram, and formulate the signaling cost and the packet delivery cost, that consists of forwarding and loss cost, to compare the proposed scheme with FMIPv6 and MIPv6. In addition, we investigate the impact of incorrect handover prediction on these costs as well.

The remainder of this paper is organized as follows. In the next section, we investigate the characteristics of MIPv6 handover and extensions for the handover latency reduction. In section 3. we present the proposed handover scheme to reduce the handover latency without handover prediction. We give numerical analysis and numerical results in section 4 and 5 respectively. Section [6] concludes this paper.

\section{Related Work}

A successful handover of an $\mathrm{MH}$ from one point of attachment to another includes new link establishment(L2 handover), IP connectivity establishment, and new route establishment when the handover leads to subnet change (L3 handover). 
We show an example of L2 and L3 handover in Fig. 1, As shown in Fig. 1 L2 handover does not always go with L3 handover. For example, L2 handover from SA 1 to SA 2 does not follow L3 handover, since SA 1 and SA 2 are in the same subnet A. However, L2 handover from SA 2 to SA 3 follows L3 handover, since the subnet of SA 2 differs from that of SA 3.

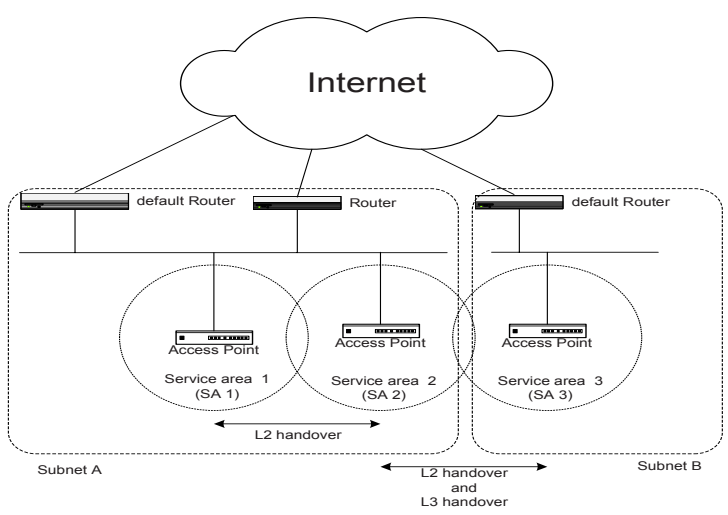

Fig. 1. An example of L2 and L3 handover

The MIPv6 is capable of handling L3 handover between different subnets, in a transparent manner for upper-layer protocol sessions. However, since L3 handover almost inevitably affects the transport protocol performance in terms of latency, mechanisms to smoothen handover, such as FMIPv6 and HMIPv6, are of interest.

For movement detection in MIPv6, an MH detects its movement by missing RA messages from the configured default router (RA assisted L3 handover) as shown in Fig. 2] (b) [1]. Therefore, an MH must wait until it receives an RA message to know the presence of a new access router (NAR) and decides that it loses reachability with the configured default router.

After detecting L3 handover, an MH performs DAD for link-local address, selects a new router, performs a prefix discovery for new router to form a new CoA and performs binding update and route optimization. Fig. [2]shows handover procedure of both RA and RS assisted L3 handover. Handover procedure can be partitioned into three steps; movement detection, address configuration, and location update.

The movement detection time $\left(t_{M D}\right)$ is the interval from when an $\mathrm{MH}$ is under the coverage of an NAR to the instant it detects its movement to the NAR by receiving an RA message from the NAR. When an MH is under the coverage of new network, it can detect its movement by an RA response to an RS message from the MH as shown in Fig. 2(a). In this case, RFC2461 specifies that RA response to RS messages must be randomly delayed by 0-500 msec. This may be a burden to provide real-time communications. 
Alternatively, it may wait for an RA message. According to the latest specification, to reduce movement detection latency in MIPv6 environments, the RA interval can be a minimum of $30 \mathrm{msec}$ to a maximum of $70 \mathrm{msec}$ [1]. However, this reduced interval leads to bandwidth consumption of a wireless link.

The address configuration time $\left(t_{A C}\right)$ is the interval from the time an $\mathrm{MH}$ detects its movement to the time it assigns its interface with a new CoA based on the prefix of the NAR. In this step, an MH generates a new CoA and performs DAD procedure. According to RFC 2461, an MH should wait 1 sec to a minimum to determine if its new CoA does not conflict with others. It is another obstacle to real-time service provision in the MIPv6 environment.

The location update time $\left(t_{U}\right)$ is the interval from the instant an MH sends a binding update message to the home agent (HA) or the correspondent node $(\mathrm{CN})$ to the instant it receives the first packet at the new attachment point. In general, the handover latency consists of $t_{M D}, t_{A C}$, and $t_{U}$.

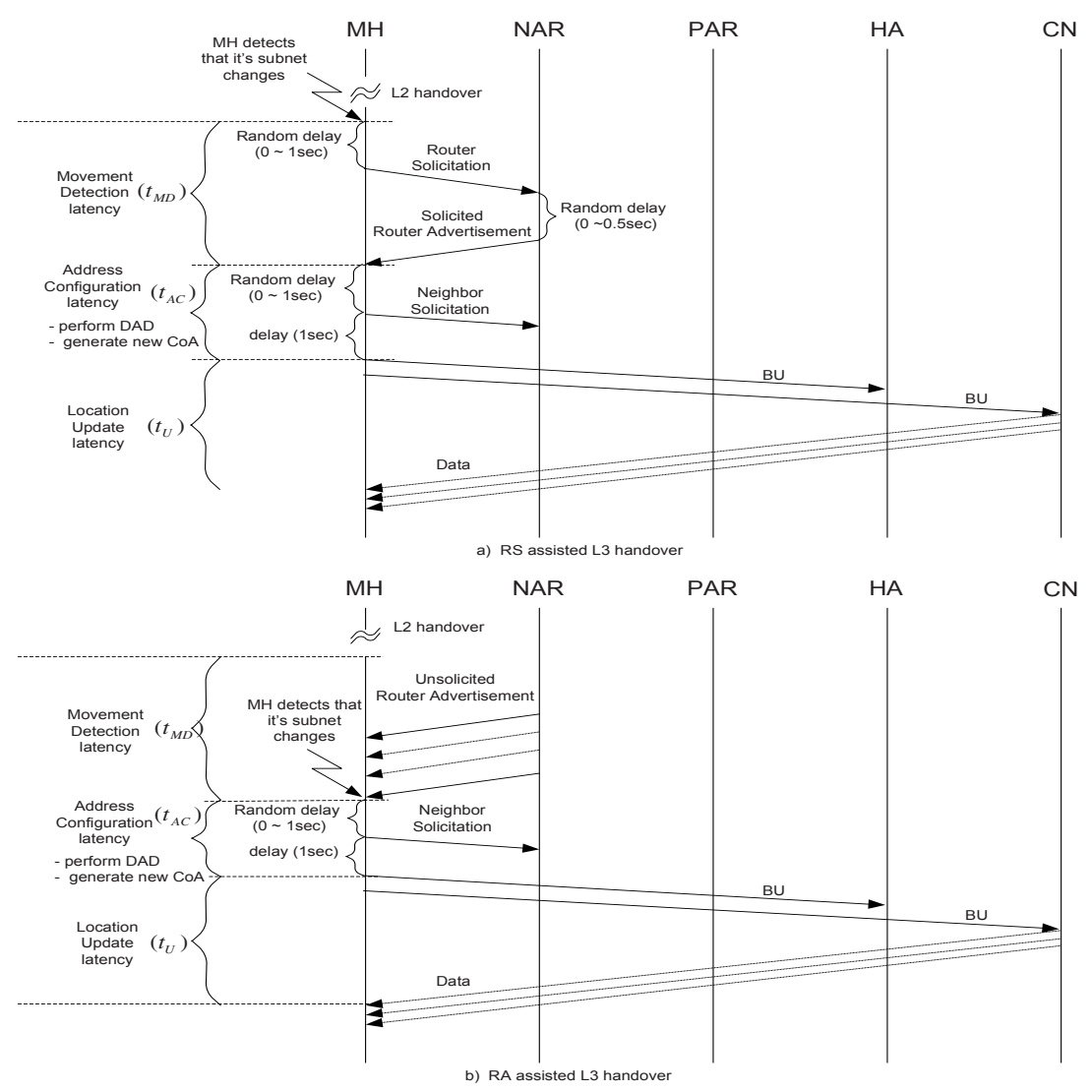

Fig. 2. L3 handover procedure in the MIPv6 environment 
FMIPv6 allows an MH to predict its attachment with a prospective default router behind a new link, by helping to prepare new IP address configuration in advance. FMIPv6 assumes that this new IP address configuration is to be received through the currently used network interface and FMIPv6 requires adding additional support to IPv6 implementation in routers, which already deployed IPv6 infrastructure may not be ready to afford.

The handover prediction executed in FMIPv6 bears the conceptual uncertainty. As neither the exact moment of L2 roaming nor the definite next AP can be foreseen and even flickering may occur, extra damage is done to the traffic, which may be larger in the case of misleading anticipation. The predictions in FMIPv6 are classified into temporal (as it foresees the moment of start and completion of the handover) and spatial (as it predicts on where the handover will lead). Both predictions may be wrong; the temporal prediction can be too early or too late or an $\mathrm{MH}$ decides to move into a different subnet from the predicted one or not to move at all.

Two recent proposals that provide movement detection optimization are a Fast Router Advertisement (FastRA) [4] and a Router Advertisement caching (FastRD) on wireless APs [5]. In a FastRD scheme, an AP scans L2 frame for an unsolicited RA message and stores it, and sends cached RA to a new $\mathrm{MH}$ as soon as L2 handover finishes. However, since an unsolicited RA message is not periodic [6], the AP should scan every incoming L2 frame to look up an unsolicited RA message. It may bring about too much cost. Our proposed scheme is similar to the FastRD scheme in terms of RA caching. However, our scheme does not need to scan every L2 frame and can be used in the case when multiple routers are on the link, while FastRD scheme does not consider the case when multiple routers are on the link. Both FastRA and FastRD are concerned with only movement detection. In both FastRA and FastRD, fast DAD procedure is required. On the contrary, our proposed scheme performs movement detection and DAD procedure are performed at once to obtain low handover latency.

In this paper, we present a non-predictive handover scheme to reduce handover latency with fewer signaling than FMIPv6. In the next section, we will describe the proposed scheme in detail.

\section{Proposed Scheme}

From the analysis of handover procedures, movement detection and DAD procedures occupy a major part of the handover latency. Our proposed scheme aims at performing movement detection and DAD procedures quickly without handover prediction. Our proposed scheme, to accelerate L3 handover procedure, uses L2 events corresponding to establishment and release of L2 association between an $\mathrm{MH}$ and an AP. Nevertheless, we do not use any event that predicts L2 handover unlike FMIPv6.

In this paper, we assume that an $\mathrm{MH}$ generates its link-local address and a new CoA using an appropriate network prefix and its interface identifier such as link-layer address. In such a case, if link-layer address of an $\mathrm{MH}$ is ascertained 
unique on the link, we can say that link-local address and new CoA of the MH are unique on the link. In the reverse order, given a network prefix and a linklayer address, the link-local address and the CoA can be generated easily. We apply this fact to our proposed scheme.

Fig. 3 shows the proposed architecture and non-predictive handover scheme to perform movement detection and DAD procedure swiftly in MIPv6 environment. In the proposed scheme, we introduce an FHA as an assistant to perform movement detection and DAD procedures quickly. As shown in Fig. 3 movement detection and DAD procedures are performed at once.

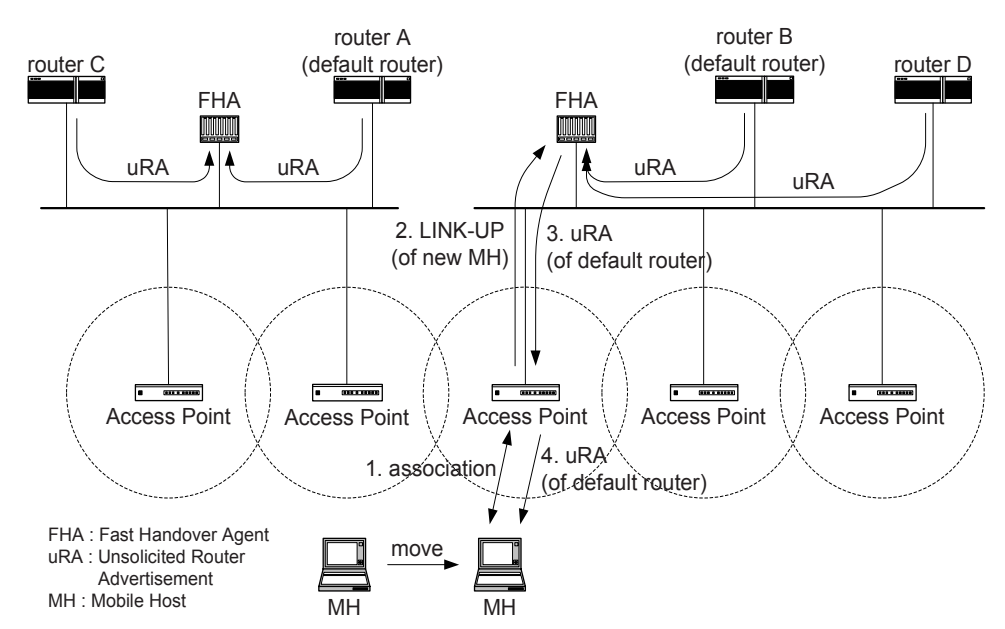

Fig. 3. Proposed architecture and non-predictive handover scheme

In the proposed scheme, FHA-based RA caching mechanism is used for default router discovery. Based on IPv6 specification, every router advertises its presence with an RA message to all node multicast address group. Therefore, the FHA receives all unsolicited RA messages from routers on the link and keeps the most recent default router's advertisement in its RA cache.

To speed up DAD procedure, we use a neighbor list in the FHA. The neighbor list stores link-layer addresses or link-local addresses of neighbors on the link. The FHA performs DAD procedure of its own accord by comparison the linklocal address generated by using link-layer address of an $\mathrm{MH}$ with the contents of the neighbor list, whenever MH's handover is notified to the FHA. In addition, there may be fixed hosts on the link. Thus the FHA should have link-local addresses of fixed hosts as well as MHs in order to perform DAD procedure internally. Since fixed nodes perform DAD procedure using Neighbor Solicitation (NS) message when they detect the attachment to the network [6], the FHA performs filtering of NS messages for DAD procedure on the link and updates the neighbor list with link-local address or discards filtered message. 
The filtering scheme of NS messages for DAD procedure is as follows. IPv6 hosts send an NS message to solicited-node multicast address group on the link. Since the solicited-node multicast address has the prefix FF:02::1:FE/104 concatenated with 24 low-order bits of IPv6 unicast address, and the NS message for DAD procedure does not include a link-layer address of the sender, the FHA can obtain NS messages for DAD procedure by filtering packets whose destination is the solicited-node multicast group and whose link-layer address option is empty.

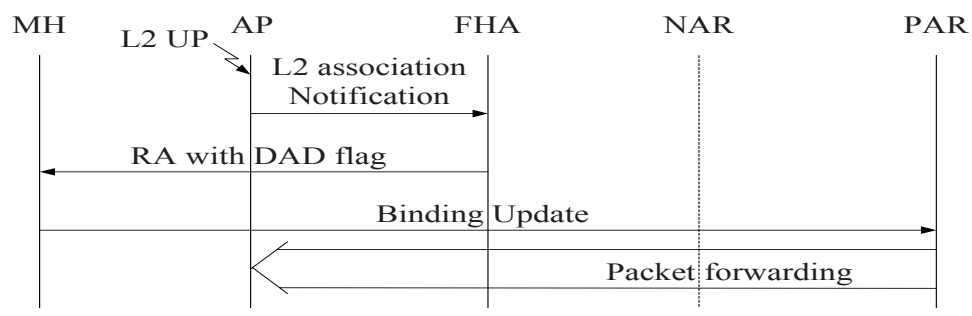

Fig. 4. The message interaction of proposed scheme

Fig. 3 and 4 represent message flows of the proposed handover scheme. Whenever an AP detects L2 handover of new MH (step 1), the AP notifies new MH's L2 handover to the FHA with LINK-UP event containing MH's link-layer address and previous AP's address (step 2). The FHA, upon receiving a notification of new MH's L2 handover, sends the cached advertisement of a default router to the MH (step $3 \& 4$ ). Then the MH uses this advertisement as a means of movement detection and DAD procedure. Based on the above procedure, the $\mathrm{MH}$, upon finishing L2 handover, can discover the default router promptly by receiving an RA message and can decide whether its subnet is changed or not. To indicate whether MH's new CoA conflicts with others by using an RA message, we define a DAD flag (D bit) in the RA message as shown in Fig. 5 If the MH's new CoA conflicts with others, D bit is set to 1 , otherwise, D bit is set to 0 . When the MH knows that its new CoA does not conflict with others, it sends immediately binding update message to the PAR in order to receive buffered packets at the PAR.

\begin{tabular}{|c|c|c|c|c|c|}
\hline Type & \multicolumn{3}{|c|}{ Code } & Check sum \\
\hline $\begin{array}{c}\text { Cur Hop } \\
\text { Limit }\end{array}$ & M & O & D & reserved & $\begin{array}{c}\text { Router } \\
\text { Lifetime }\end{array}$ \\
\hline \multicolumn{4}{|c|}{ Reachable Time } \\
\hline
\end{tabular}

Fig. 5. Modified RA message for DAD procedure 
When the proposed scheme is applied to DAD procedure, there is a problem in that neighbors cached in the FHA's neighbor list may differ from actual neighbors on the link. This situation comes from the fact that DAD procedure is performed only when the host configures its network interface with newly generated address and there is no mechanism to notify moving out the link or disconnection of a host to the FHA.

In our proposed scheme, to maintain more accurate information on neighbors on the link in the FHA's neighbor list, the FHA performs periodically Neighbor Unreachable Discovery (NUD) to the fixed hosts in the neighbor list and updates the state.

\section{Performance Analysis}

In this section, we analyze the proposed scheme, MIPv6, and FMIPv6 in terms of handover latency, signaling cost, and packet delivery cost. We don't compare the proposed scheme with FastRD and FastRA, since both FastRA and FastRD are concerned with only movement detection optimization. For both FastRA and FastRD, fast DAD procedure is required.

\subsection{Handover Latency}

With handover latency, we specify three latencies such as link switching latency 1 , IP connectivity latency, and location update latency similar to [10. The link switching latency $\left(t_{L}\right)$ is due to L2 handover. The IP connectivity latency $\left(t_{I}\right)$ is due to new IP address configuration and movement detection after L2 handover. an $\mathrm{MH}$ can send packets from a new subnet link after IP connectivity latency. The location update latency $\left(t_{U}\right)$ is the latency in forwarding IP packets to MH's new IP address.

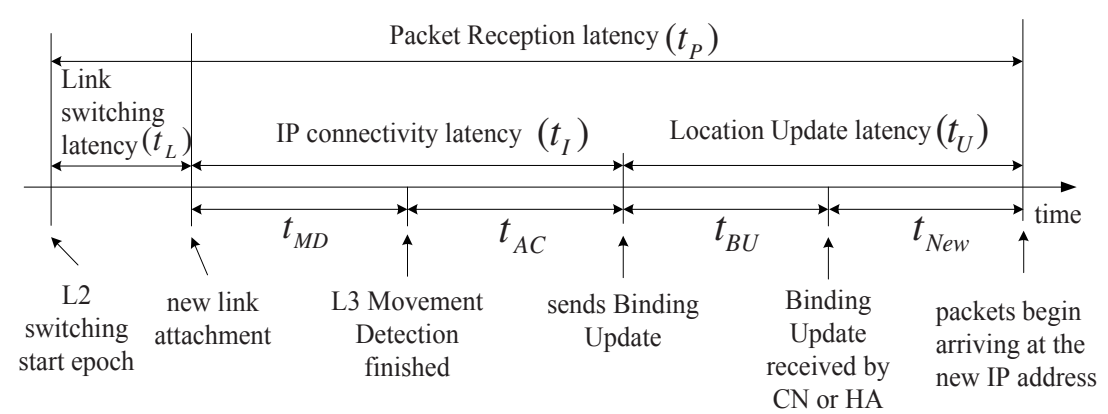

Fig. 6. Timing diagram in basic MIPv6

In discussion on handover latency, we consider two scopes; when an $\mathrm{MH}$ sends packets with new IP address and when the MH receives packet after L2

\footnotetext{
${ }^{1}$ link switching latency is the same as L2 handover latency.
} 
handover. The IP connectivity latency reflects how quickly an MH can send IP packets after L2 handover. To consider how quickly an $\mathrm{MH}$ can receive IP packets after L2 handover, we specify the packet reception latency $\left(t_{P}\right)$, the period from the starting point of L2 handover to when an $\mathrm{MH}$ receives packets for the first time after L2 handover. To represent the $t_{P}$ and $t_{I}$, we define the round-trip delay $\left(t_{M N}\right)$ between an $\mathrm{MH}$ and an NAR, the round-trip delay $\left(t_{N P}\right)$ between an NAR and a PAR, the round-trip delay $\left(t_{A F}\right)$ between an AP and an FHA, and the round-trip delay $\left(t_{M F}\right)$ between an MH and an FHA.

Fig. 6 shows the timing diagram corresponding to basic MIPvd 2 (B-MIPv6). In basic MIPv6, the packet reception latency is $t_{L}+t_{I}+t_{U}$ and the IP connectivity latency is $t_{M D}+t_{A C}$.

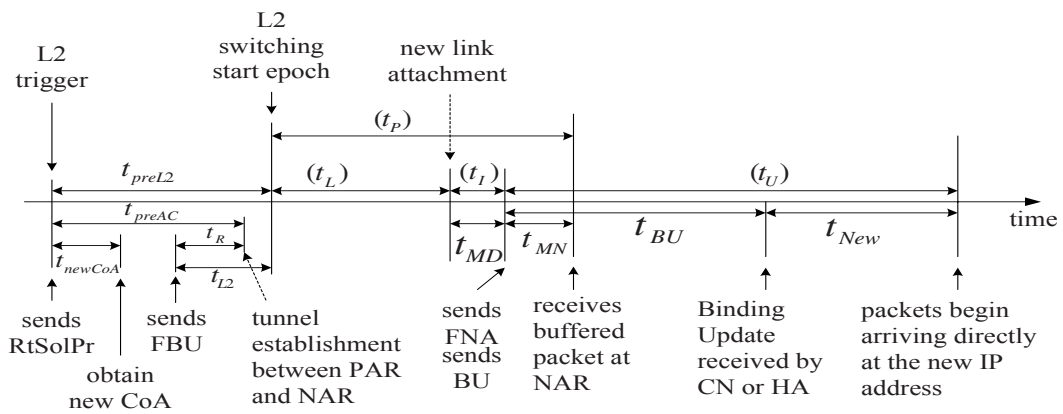

(a) predictive FMIPv6

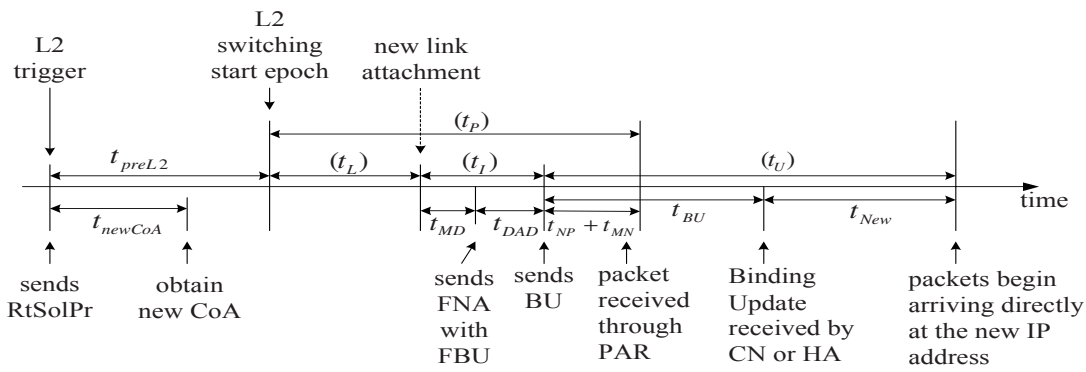

(b) reactive FMIPv6

Fig. 7. Timing diagram in FMIPv6

In Fig. 7 (a) represents the timing diagram of predictive FMIPv6 (PFMIPv6) and (b) represents that of reactive FMIPv6 (R-FMIPv6). In predictive FMIPv6, an MH can send packets as soon as movement detection, since the address configuration is finished before L2 handover finishes. In addition, packets buffered at the NAR are forwarded to an MH as soon as the NAR receives an FNA message from an MH. In R-FMIPv6, an MH can send packets after

\footnotetext{
${ }^{2}$ We represent MIPv6 as basic MIPv6 after this.
} 
movement detection and DAD procedures, and can receive packets when the PAR forwards packets to an MH after it receives MH's FBU message. The IP connectivity latencies of P-FMIPv6 and R-FMIPv6 are $t_{M D}$ and $t_{M D}+t_{D A D}$, respectively. The packet reception latencies of P-FMIPv6 and R-FMIPv6 are $t_{L}+t_{I}+t_{M N}$ and $t_{L}+t_{I}+t_{M N}+t_{N P}$, respectively.

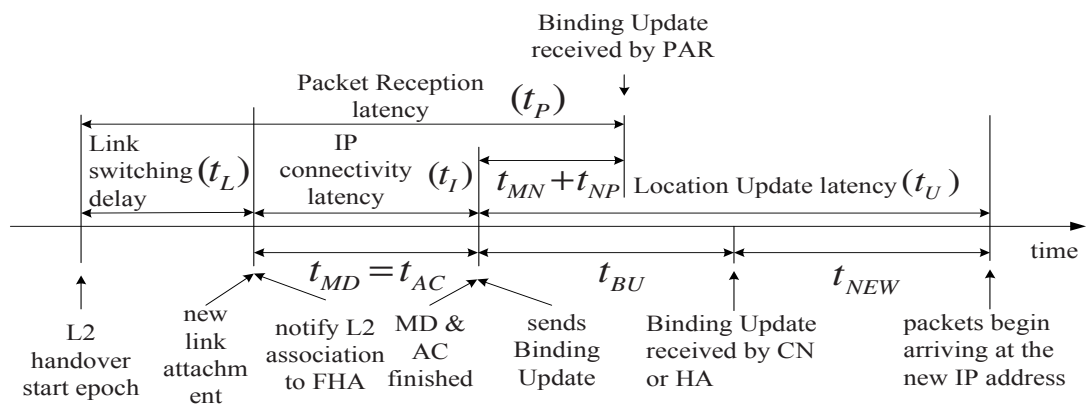

Fig. 8. Timing diagram in proposed scheme

Fig. 8 shows the timing diagram corresponding to the proposed scheme. The proposed scheme improves IP connectivity latency preponderantly as described in the previous section. The IP connectivity latency includes the transmission delay from an AP to an FHA and the transmission delay from an FHA to an $\mathrm{MH}$. The IP connectivity latency is $\frac{t_{A F}}{2}+\frac{t_{M F}}{2}$ and the packet reception latency is $t_{L}+t_{I}+t_{M N}+t_{N P}$.

\subsection{Signaling Cost}

In this section, we analyze signaling cost incurred in FMIPv6, B-MIPv6, and the proposed scheme. Similar to [7], we define transmission cost between nodes and processing cost at the nodes to be used in the rest of this paper. The $T C_{M P}$, $T C_{M N}$, and $T C_{M F}$ are transmission costs incurred in the wireless link between an $\mathrm{MH}$ and a PAR, between an $\mathrm{MH}$ and a NAR, and between an $\mathrm{MH}$ and an FHA, respectively. The $T C_{N P}$ is transmission cost incurred in the wired link between a NAR and a PAR. The $T C_{A F}$ is transmission cost incurred in the wired link between an $\mathrm{AP}$ and an FHA. The $a_{P}, a_{N}$, and $a_{F H A}$ are processing costs at the PAR, the NAR, and the FHA, respectively.

We denote signaling cost of B-MIPv6, P-FMIPv6, R-FMIPv6, and the proposed scheme as $C S_{\text {basic }}, C S_{\text {pred }}, C S_{\text {react }}$, and $C S_{\text {prop }}$, respectively. In this analysis, we assume that movement detection is accomplished by receiving an RA message in B-MIPv6 and movement detection is accomplished by RS/RA message exchanges in FMIPv6. In addition, we exclude the location update cost from signaling cost.

We assume that transmission cost is proportional to the distance between the source and the destination and proportionality constant is $\delta_{C}$. In addition, 
we assume that the transmission cost over the wireless link is $\rho$ times higher than the unit distance wireline transmission cost, $\theta_{S}$. For example, $T C_{N P}$ can be expressed as $T C_{N P}=l_{N P} \theta_{S} \delta_{C}$, where $l_{N P}$ is an average distance between a NAR and a PAR, and $T C_{M N}$ can be expressed as $T C_{M N}=\rho \theta_{S} \delta_{C}$, where the average distance $l_{M N}$ between an $\mathrm{MH}$ and a NAR is 1 . Using the proportionality constant $\delta_{C}$ and weighting factor $\rho$ for the wireless link, each signaling cost can be rewritten as follows:

$$
\begin{aligned}
C S_{\text {basic }} & =2 T C_{M N}=2 \rho \theta_{S} \delta_{C} \\
C S_{\text {pred }} & =4 T C_{M P}+3 T C_{N P}+3 T C_{M N}+3 a_{P}+2 a_{N} \\
& =\left(7 \rho+3 l_{N P}\right) \theta_{S} \delta_{C}+3 a_{P}+2 a_{N} \\
C S_{\text {react }} & =2 T C_{M P}+2 T C_{N P}+3 T C_{M N}+2 a_{P}+a_{N} \\
& =\left(5 \rho+2 l_{N P}\right) \theta_{S} \delta_{C}+2 a_{P}+a_{N} \\
C S_{\text {prop }} & =T C_{A F}+T C_{M F}+T C_{M N}+T C_{N P}+a_{F H A}+a_{P} \\
& =\left(2 \rho+l_{A F}+l_{N P}\right) \theta_{S} \delta_{C}+a_{F H A}+a_{P}
\end{aligned}
$$

\subsection{Packet Delivery Cost}

With packet delivery cost, we consider the cost associated with both forwarding packets (forwarding cost) and lost packets (loss cost). In this analysis, we consider the forwarding cost as the additional buffer space used by forwarding packets during the handover period. Packet delivery cost consisting of forwarding cost and loss cost is defined as $\alpha \cdot C_{\text {forwarding }}+\beta \cdot C_{\text {loss }}$, where $\alpha$ and $\beta$ are weighting factors.

In B-MIPv6, only loss cost does exist in the packet delivery cost. Consequently, given the packet arrival rate $\lambda_{p}, C P_{\text {basic }}$ can be expressed as Eq. 1] In the proposed scheme, the packet delivery cost consists of only forwarding cost. The packets, forwarded to the PAR, are buffered until MH's binding update message arrives at the PAR. As a result, the $C P_{\text {prop }}$ can be expressed as Eq. 2

In FMIPv6, packet buffering at the NAR, based on handover prediction, is supported to avoid packet loss and to give a smooth handover. However, because of wrong temporal prediction, some packets may be lost. $t_{L 2}$ denotes the time period from when the FBU message is sent to the starting point of L2 handover and $t_{R}$ denotes the time period from when the FBU message is sent to when the tunnel is established. If $t_{L 2}$ is less than $t_{R}$, packets arriving at the PAR during $\left(t_{R}-t_{L 2}\right)$ period may be lost, since the tunnel is not yet established. Thus, the loss cost can be expressed as $\lambda_{p} \beta \max \left\{\left(t_{R}-t_{L 2}\right), 0\right\}$. Consequently, the packet delivery cost in P-FMIPv6 and R-FMIPv6 can be expressed as Eq. 3 and Eq. 4 . respectively.

$$
\begin{aligned}
& C P_{\text {basic }}=\lambda_{p} \beta\left(t_{L}+t_{I}+t_{U}\right) \\
& C P_{\text {prop }}=\lambda_{p} \alpha\left(t_{L}+t_{I}+\frac{t_{M N}}{2}+\frac{t_{N P}}{2}\right)
\end{aligned}
$$




$$
\begin{aligned}
C P_{\text {pred }}= & \lambda_{p}\left[\alpha\left\{t_{L}+t_{I}+\frac{t_{M N}}{2}+\max \left\{\left(t_{L 2}-t_{R}\right), 0\right\}\right\}\right. \\
& \left.+\beta \max \left\{\left(t_{R}-t_{L 2}\right), 0\right\}\right] \\
C P_{\text {react }}= & \lambda_{p} \beta\left(t_{L}+t_{I}+\frac{t_{M N}}{2}+\frac{t_{N P}}{2}\right)
\end{aligned}
$$

In the case of a wrong spatial prediction, the forwarded packets to the wrongly predicted NAR may be lost and this packet forwarding to the wrongly predicted NAR is finished when MH's FBU message arrives at the PAR. Therefore, packet delivery cost in the wrong spatial prediction case of FMIPv6 can be expressed as Eq. 5 .

$$
C P_{\text {wrong }}=\lambda_{p} \beta\left[\left(t_{L}+t_{I}+\frac{t_{M N}}{2}+\frac{t_{N P}}{2}\right)+\max \left\{\left(t_{L 2}-t_{R}\right), 0\right\}\right]
$$

\section{$5 \quad$ Numerical Results}

In this section, we demonstrate some numerical results. For the numerical analysis of handover latency and signaling cost, we set the values of parameters as shown in Table 1. These values are based on the empirical results of 8 and reference values defined in [79|10].

Table 1. Parameters for numerical analysis

\begin{tabular}{|c|c|c|c|c|c|c|c|c|c|c|c|c|c|c|c|c|}
\hline \multicolumn{1}{|c|}{ latency analysis (in msec) } & \multicolumn{10}{|c|}{ signaling cost analysis } \\
\hline$t_{L}$ & $t_{M N}$ & $t_{N P}$ & $t_{A F}$ & $t_{M F}$ & $t_{R}$ & $a_{N}$ & $a_{P}$ & $a_{F H A}$ & $\delta_{C}$ & $l_{A F}$ & $l_{N P}$ & $\rho$ & $\theta_{S}$ & $\lambda_{p}$ & $\alpha$ & $\beta$ \\
\hline 50 & 14 & 6 & 6 & 14 & 20 & 5 & 5 & 10 & 0.1 & 1 & 5 & 10 & 1 & 1 & 0.2 & 0.8 \\
\hline
\end{tabular}

In B-MIPv6 case, an MH does not use L2 trigger events and detects its movement by RA beacon message. the $\mathrm{MH}$ can detect its movement within 27 msec on average, and within $70 \mathrm{msec}$ at worst [11]. In addition, an MH waits 1 sec after sending an NS message for DAD with random delay, to confirm that its address does not conflict with others [6]. Consequently, average $t_{I}$ and $t_{P}$ in B-MIPv6 are about $1527 \mathrm{msec}$ and $1677 \mathrm{msec}$, respectively. In FMIPv6, an $\mathrm{MH}$ detects its movement by RS/RA message exchanges triggered by L2 events. Therefore, $t_{M D}$ is $14 \mathrm{msec}$. In P-FMIPv6, $t_{I}$ and $t_{P}$ are $14 \mathrm{msec}$, and 78 msec, respectively. In R-FMIPv6, minimum $t_{I}$ and $t_{P}$ are $28 \mathrm{msec}$ and $98 \mathrm{msec}$, respectively. However, if normal DAD procedure is applied to R-FMIPv6, 1 sec latency should be added to previous minimum values. In the proposed scheme, $t_{I}$ is $10 \mathrm{msec}$ and $t_{P}$ is $83 \mathrm{msec}$. In terms of the IP connectivity latency, the proposed scheme gives the lowest latency, since movement detection and DAD procedures are performed at once. From the numerical results, we found that proposed scheme gives lower latency as well as fewer signaling messages than P-FMIPv6 in terms of the IP connectivity. Finally, we can conclude that our scheme can 


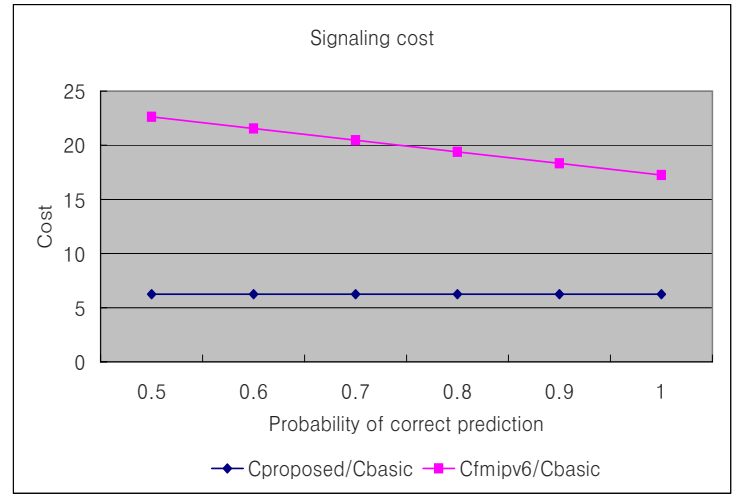

(a) Signaling cost

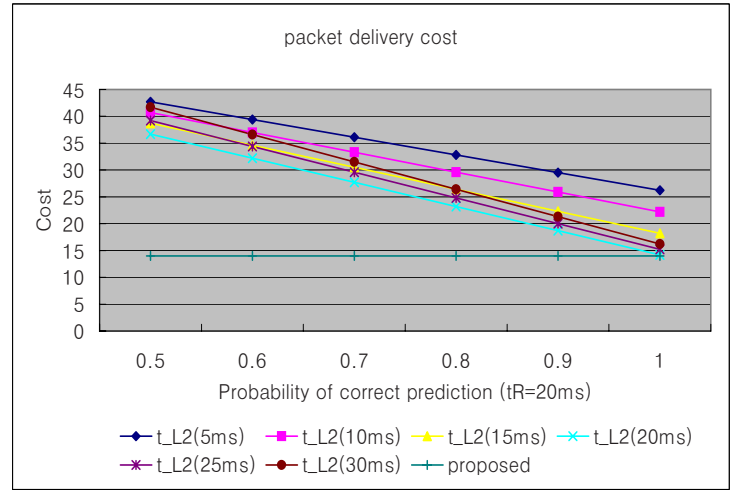

(b) Packet delivery cost

Fig. 9. Signaling and packet delivery costs

be used to support real-time applications in mobile IPv6 environment without handover prediction.

An additional signaling cost of FMIPv6 in the wrong spatial prediction case is $\left(3 \rho+2 l_{N P}\right) \theta_{S} \delta_{C}+3 a_{P}+2 a_{N}$. Let $P_{s}$ be the probability of prediction accuracy. Then, the signaling cost of FMIPv6 can be expressed as shown in Eq. 6] Similar to the signaling cost of FMIPv6, the packet delivery cost of FMIPv6, $C P_{f m i p}$, can be expressed as a function of the probability of prediction accuracy as shown in Eq. 7.

$$
\begin{aligned}
C S_{\text {fmip }}= & P_{s} \cdot C S_{\text {pred }}+\left(1-P_{s}\right)\left(C S_{\text {wrong }}+C S_{\text {react }}\right) \\
& \text { where } C S_{\text {wrong }}=\left(3 \rho+2 l_{N P}\right) \theta_{S} \delta_{C}+3 a_{P}+2 a_{N} .
\end{aligned}
$$




$$
C P_{\text {fmip }}=P_{s} \cdot C P_{\text {pred }}+\left(1-P_{s}\right) \cdot C P_{\text {wrong }}
$$

The signaling costs of B-MIPv6, the proposed scheme and FMIPv6 (when $\left.P_{s}=100 \%\right)$ are $2,12.5$, and 34.5 , respectively. The reason why the signaling cost of B-MIPv6 is much smaller than others is there is no binding update signaling to the PAR after L2 handover.

Fig. 9(a) shows the relative signaling cost as $P_{s}$ changes. The more incorrect the handover prediction, the more the signaling overhead in FMIPv6 rises. In addition, Fig. 9(b) shows the packet delivery cost as $P_{s}$ and $t_{L 2}$ change. As shown in Fig. 9(b), when the probability of incorrect prediction is zero, the packet delivery cost of the proposed scheme is similar to that of FMIPv6. When the probability of incorrect prediction is not zero, the packet delivery cost of FMIPv6 is higher than that of ours. This result comes from the packet loss caused by incorrect handover prediction.

From Fig. 9, we conclude that incorrect handover prediction in FMIPv6 brings on additional overhead in terms of signaling and packet loss.

\section{Conclusion}

In this paper, we propose a low-latency non-predictive handover scheme to reduce handover latency in basic MIPv6 and analyze the impact of incorrect prediction in FMIPv6 on mobility management in terms of signaling cost and packet delivery cost. In addition, we compare handover latency of the proposed scheme, FMIPv6, and basic MIPv6 by using timing diagram. Although the proposed scheme does not predict handover, it reduces handover latency with fewer signaling messages than FMIPv6 and gives low packet delivery cost as well. The reasons why the proposed scheme gives low handover latency are that movement detection and DAD procedures are performed at once using FHA-based RA caching and neighbor list and movement detection is triggered by LINK-UP event.

In future work, we plan to extend the proposed scheme with the cases where the hierarchical MIPv6 scheme does exist. In addition, we plan to simulate the proposed scheme under several scenarios where MHs move with different velocities, incorrect movement predictions are abundant, and the movement to the same link is generated at the same time.

\section{References}

1. D. Johnson and C. Perkins, Mobility support in IPv6, IETF drft, March 2002.

2. H. Soliman et al., Hierarchial MIPv6 Mobility Management IETF draft, July 2002.

3. G. Dommety et al., Fast Handover for Mobile IPv6, IETF draft, March 2002.

4. J. Kempf et al., IPv6 Fast Router Advertisement, IETF draft, Oct. 2003.

5. J. H. Choi et al., Fast Router Discovery with RA caching in AP, IETF draft, Feb. 2003 .

6. T. Narten et al., RFC 2461 Neighbor Discovery for IP Version 6, 1998. 
7. J. Xie and F. Akyildiz, A Novel Distributed Dynamic Location Management Scheme for Minimizing Signaling Costs in Mobile IP, IEEE Trans. on Mobile Computing, 2002.

8. Mishra et al., An Emprical Analysis of the IEEE 802.11 MAC Layer Handff Process, ACM SIGCOMM CCR, Oct. 2003.

9. S. Park and Y. Choi, Performance Analysis of Fast Handover in Mobile IPv6 Networks, IFIP PWC 2003, 2003.

10. R. Koodli and C. Perkins, Fast Handovers and Context Transfers in Mobile Networks, ACM SIGCOMM CCR, Oct. 2001.

11. G. Daley, B. Pentland, and R. Nelson, Movement Detection Optimizations in Mobile IPv6, ICON 2003, 2003. 